\title{
Emission-line-profile variability as a contraint on the structure and dynamics of hot star winds
}

\author{
Luc Dessart \\ Sterrekundig Instituut, Universiteit Utrecht, \\ Princetonplein 5, NL-3584 CC Utrecht, Nederland \\ Stanley P.' Owocki \\ Bartol Research Institute, University of Delaware, \\ Newark, DE 19716, USA
}

\begin{abstract}
We present theoretical calculations of emission-line-profile variability (LPV), based on radiation hydrodynamics simulations of the infamous radiative instability of hot star winds. We demonstrate that spherically symmetric wind structures (shells) cannot account for the observed profile variability at line center. Hence, we resort to a model that breaks-up the wind volume into a number of independent star-centered cones. The essential approximation made here is that each of these cones can be described by a structure calculated with a one-dimensional (1D) radiation hydrodynamics model. Such pseudo-3D 'patch'method leads to a satisfactory reproduction of the fundamental characteristics of LPV observed in O-type and Wolf-Rayet star optical spectra: the low-level fluctuations in the profile centre region, a migration of variable sub-peaks from line center to edge, that mimics the underlying wind acceleration. Our method highlights the correlation between the velocity scale of profile sub-peaks at line center and the lateral extent of wind structures, while at line edge it reflects the intrinsic radial velocity dispersion of emitting clumps. However, our model fails to reproduce the increase in this characteristic velocity scale from line center to edge, which we believe is a shortcoming of our purely 1D hydrodynamics approach.
\end{abstract}

\section{Introduction}

Hot star winds are believed to be driven by scattering and absorption of the continuum star light by optically thick lines of ions. This radiative driving mechanism is known to be highly unstable (Owocki \& Rybicki 1984), transforming the smooth wind lay-out predicted by Castor, Abbott \& Klein (1975) into a structured outflow, made-up of strongly accelerated rarefied regions and dense, slow and radially confined knots (Owocki, Castor \& Rybicki 1988). Our study investigates whether such radiative-instability generated structures are at the origin of the observed emission-line-profile variability (LPV) discussed in Robert (1992) and Lépine \& Moffat (1999), which typically shows a deviation from the mean profile of $\sim 1 \%$, distinct profile sub-peaks migrating away from line centre to the nearest line edge, with a concomitant increase in their characteristic velocity scale $\left(50-200 \mathrm{~km} \mathrm{~s}^{-1}\right)$. 


\section{Theoretical investigations}

Based on radiation hydrodynamics simulations, we compute the temporal evolution of a density-square sensitive emission line. Within the assumption of spherical symmetry, we find that the variability is concentrated in the lineprofile wings, contrary to observations. But how can we break this symmetry and move to 3D while retaining the feasibility of profile modeling? Motivated by the essentially radial nature of radiative driving, we break up the 3D wind into independent and identical cones. For example, for a $1^{\circ}\left(3^{\circ}\right)$ patch, $\sim 41000$ (4600) patches are needed to cover the full solid angle. We run our 1D radiation hydrodynamics model to generate a large database of 1D wind lay-outs, randomly allocate one snapshot in the time sequence to each cone, and perform a time evolution of the wind emissivity.

LPV characteristics are satisfactorily reproduced for a $3^{\circ}$ patch and a line forming within the accelerating wind. We understand the LPV as resulting from the fluctuating average of contributions from $\sim 10^{4-5}$ radiative-instability generated wind structures within the line emission region - this depends on the size of the emission region and the clumpiness of the wind. A bigger (smaller) patch enhances (decreases) the level of variability beyond (below) its observed value (Dessart \& Owocki 2002a). We find that the sub-peak migration reflects the underlying wind acceleration, so that lines that form deeper in the wind span a bigger range of profile frequencies per unit time than outer forming lines. It is still difficult to state the steepness of the acceleration, because hot star radii are still poorly known.

LPV contains essential information on the geometry/dynamics of hot star wind structures. We find that the sub-peak broadening at line centre is controled by the lateral extent of wind structures, while the intrinsic radial velocity dispersion of wind structures is the central element at line edge (Dessart \& Owocki 2002b). A wavelet analysis on such synthetic LPV permitted a quantitative assessment of the velocity scale $\sigma_{\xi}$ of profile sub-peaks and an objective comparison with Lépine \& Moffat (1999). $\sigma_{\xi}$ is observed to increase from 50 to $200 \mathrm{~km} \mathrm{~s}^{-1}$ from centre to line edge. The line-centre value is reproduced with a wind structure lateral size of $\sim 3^{\circ}$, but we fail to reproduce the observed magnitude of radial velocity dispersion intrinsic to wind blobs, a possible artifact of 1D hydrodynamics simulations and their associated lack of turbulence.

\section{References}

Castor, J.I., Abbott, D.C., Klein, R.I. 1975, ApJ 195, 157

Dessart, L., Owocki, S.P. 2002a, A\&A 383, 1113

Dessart, L., Owocki, S.P. 2002b, A\&A 393, 991

Lépine, S., Moffat, A.F.J. 1999, ApJ 514, 909

Owocki, S.P., Rybicki, G.B. 1984, ApJ 284, 337

Owocki, S.P., Castor, J.I., Rybicki, G.B. 1988, ApJ 335, 914

Robert, C. 1992, $\mathrm{PhD}$ thesis, Université de Montréal, Canada 